\title{
Modelling the tuned criticality in stick-slip friction during metal cutting
}

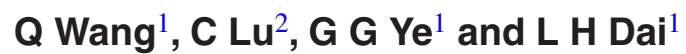 \\ ${ }^{1}$ State Key Laboratory of Nonlinear Mechanics, Institute of Mechanics, Chinese \\ Academy of Sciences, Beijing 100190, People's Republic of China \\ ${ }^{2}$ Department of Mechanical Engineering, Curtin University, Perth, WA 6845, \\ Australia \\ E-mail: 1hdai@lnm.imech.ac.cn
}

Received 9 April 2015

Accepted for publication 21 May 2015

Published 16 June 2015

\begin{abstract}
Cutting is a ubiquitous process in nature and man-made systems. Here we demonstrate that, based on morphological patterns observed in experiments, the friction behaviour of metal cutting exhibits a criticality with cutting speed as a tuned parameter. The corresponding stick-slip events can be described by a power law distribution. A dynamic thermo-mechanical model is developed to investigate how such a tuned criticality occurs. It is shown that, in terms of the linear stability analysis, stick-slip friction is due to the thermo-mechanical instability and dynamical interaction between shear dissipation and nonlinear friction. Moreover, there is a secondary transition from a criticality state to a limit cycle that is dominated by the inertia effect, which is similar to the frequency lock phenomenon in a forced Duffing oscillator.
\end{abstract}

Keywords: stick-slip, friction, criticality, stability analysis, metal cutting

(Some figures may appear in colour only in the online journal)

\section{Introduction}

Metal cutting is an important material processing method that has drawn attention over the past few decades [1]. Since the tool-chip friction directly affects the surface finish and tool wear, it has been extensively studied in previous works [2]. The traditional cutting theory has played a significant role in the development of machining technology [3, 4]. Due to the complexity of contact conditions, however, a full understanding of the physics behind cutting friction has not been developed. Friction in a metal cutting process involves the interaction between two contact surfaces, plastic shear stress dissipation, and mass and energy transfer. 


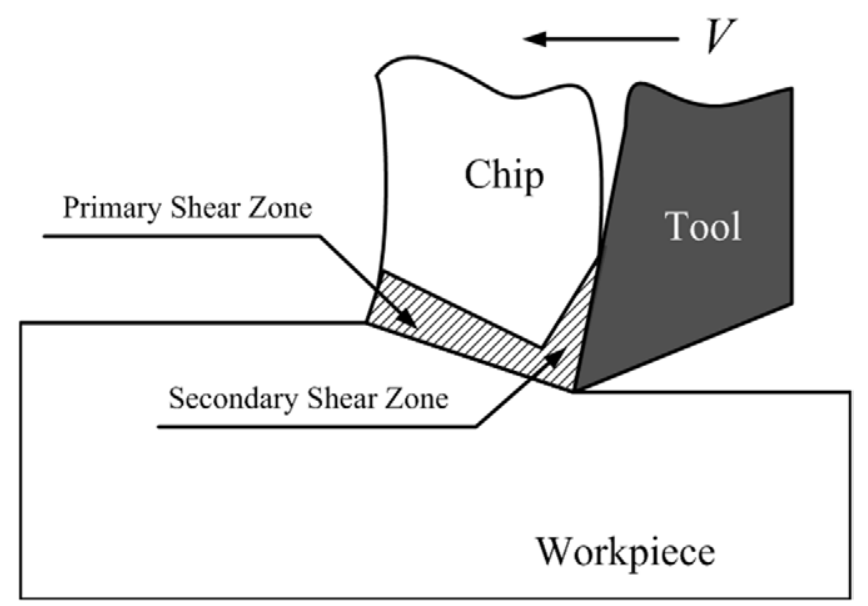

Figure 1. Schematic of a metal cutting system.

It is shown that as cutting speed increases, nonlinear phenomena emerge in a metal cutting process such as stick-slip friction [5] and friction chatter [6].

The stick-slip friction refers to a jerky motion along two contact surfaces, which is considered as a kind of friction instability [7]. Such a phenomenon is widely observed in seismic fault dynamics [8] and amorphous material deformation [9]. In metal cutting as illustrated in figure 1, there are two main nonlinear responses: the intensive shear deformation in primary shear zone (PSZ) and friction in tool-chip interface that is defined as secondary shear zone (SSZ). It is shown that, at a higher cutting speed, morphological patterns of chips change from continuous to segmented ones. This is mainly due to the repeated adiabatic shear bands in PSZ $[10,11]$. Meanwhile, the stick-slip friction also appears on the tool-chip interface in SSZ [12]. Thus, high-speed metal cutting is a multiple interaction, non-equilibrium system, strain rate effect is significant during that condition [13]. There is evidence that PSZ has an influence on SSZ [14]. In our experiments, we find out that the stick-slip traces on chips follow a power law scaling property. Under a specific speed range, friction slips in a cutting system exhibits a similar size distribution to the morphological behaviour of crack front propagation [15]. It is also shown that, acoustic emission signals in scratching superhard coatings, a process that resembles to metal cutting, yield a power law distribution [16]. These similarities imply that the metal cutting process might evolve to a criticality state due to interactions between external loading and internal energy dissipation [17]. However, the power law scaling in metal cutting is more likely a speed-tuned criticality rather than a selforganized criticality $[18,19]$.

As is well known, the critical phenomena that are frequently observed in multi-dimensional dynamical systems are plenty in nature, for example, from fibre bundles [17] at large scales to microcrack coalescence [20] at small scales. In this paper, we pay attention to morphological patterns observed in tool-chip interface friction and their statistical properties under the orthogonal cutting condition. The paper is organized as follows. In section 2, we first describe the experimental setup in details and present several typical results under different cutting speeds. Then, to elucidate the mechanism of a speed-tuned criticality in the metal cutting process, we propose a thermo-mechanical coupled dynamical model with stability analysis and numerical simulations in section 3. Finally, a comparison with previous studies is discussed and the conclusion is given in section 4 . 


\section{Experiment}

\subsection{Experimental setup}

Experiments were carried out on a split Hopkinson pressure bar. By using a specially designed device, the cutting speed can change from 3 to $13 \mathrm{~m} \mathrm{~s}^{-1}$ [21].

As illustrated in figure 2(a), the cutting tool (uncoated P10 carbide) is fixed on the projectile. The tool rake angle is $0^{\circ}$ and the clearance angle is $5^{\circ}$. After cutting, the tool hits a transmitter bar and the latter is stopped by a shock absorber. The cutting speed was measured by using laser detectors and an electronic time counter. Ti-Al6-V4, a typical hard machining material, was used as the specimen with the form of a rectangular plate made by electrical discharge machining, as seen in figure 2(b). During the orthogonal cutting, the same depth of $100 \mu \mathrm{m}$ was controlled with different driving velocities because our focus is on the morphology evolution of chips. In the case of a lower machining speed between 0.03 and $3 \mathrm{~m} \mathrm{~s}^{-1}$, an ordinary lathe was applied with the same material and cutting tool.

\subsection{Experimental results}

By using a high-resolution scanning electron microscopy, morphologies of collected chips were examined on their free surfaces and tool-chip frictional interfaces. As shown in figure 3, these patterns transfer from steady sliding to stick-slip as the cutting speed increases.

In the case of a cutting speed lower than $1 \mathrm{~m} \mathrm{~s}^{-1}$, the tool-chip interface is relatively smooth; while in a higher cutting velocity (see figure 3(c)), a typical stick-slip pattern can be observed. It implies that the cutting friction process is intermittent, with a slow build-up and then an avalanche. The slip-length becomes regular as the cutting speed increases. In figure 3(e), it is seen that the shear displacement on front chip surface is roughly the same as the stick-slip frictional trace at tool-chip interface. These synchronous patterns indicate that the strong mutual influence exists between shear bands and friction in metal cutting. It is such an interaction that plays a key role in the occurrence of a speed-tuned criticality during metal cutting.

Since the magnitude of a stress drop can be characterized by its slip-length in a stick-slip process, statistical analysis was performed on the slip-length distributions at different cutting speeds. Here, the slip length was determined by the size of a smooth area left by fast sliding of cutting chips after a stick stage (see figure 3 ).

As shown in figure 4, there are four groups based on their slip-length distributions. It is obvious that, at a lower cutting speed of $3 \mathrm{~m} \mathrm{~s}^{-1}$, there is a decreasing, long-tail distribution. As the cutting speed increases, however, it gradually turns into a positively skewed distribution at $5 \mathrm{~m} \mathrm{~s}^{-1}$, and then a normal distribution at $10 \mathrm{~m} \mathrm{~s}^{-1}$. To avoid statistical fluctuations induced by arbitrarily chosen segments, a cumulative probability distribution was adopted in data analysis.

By fitting the data as shown in figure 5 , the cumulative probability of slip events at $3 \mathrm{~m} \mathrm{~s}^{-1}$ can be described by a power law with an exponential cutoff, that is

$$
P(>s) \infty s^{-\gamma} \exp \left(-s / s^{*}\right),
$$

where $s$ is the slip-length, $s^{*}=104.4 \pm 1.9 \mu \mathrm{m}$ is the characteristic cutoff length, and $\gamma=$ $0.44 \pm 0.02$ is the power exponent. In contrast, in the case of $10 \mathrm{~m} \mathrm{~s}^{-1}$, the cumulative distribution can be well fitted by an exponential function

$$
P(>s) \infty \exp \left(-\left(s / s^{*}\right)^{2}\right),
$$

with the characteristic (or average) slip-length $s^{*}=74.5 \pm 0.7 \mu \mathrm{m}$. 
(a) Launch tube Laser detectors Cutting specimen

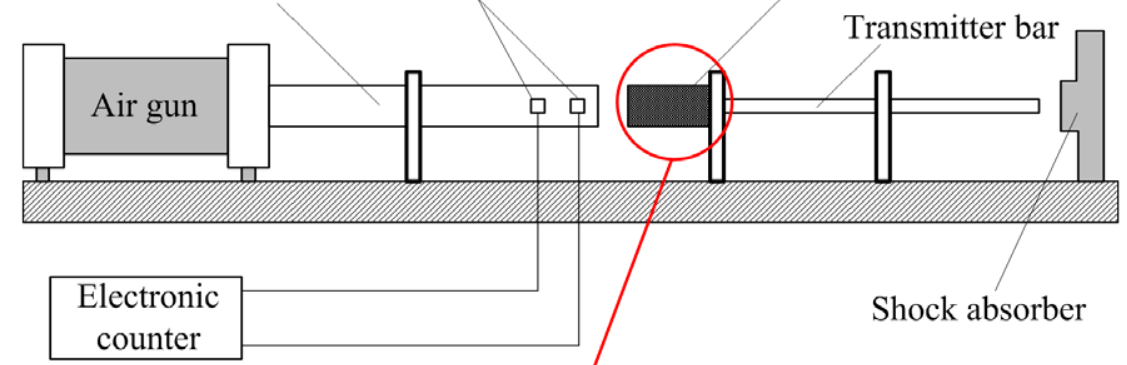

(b)

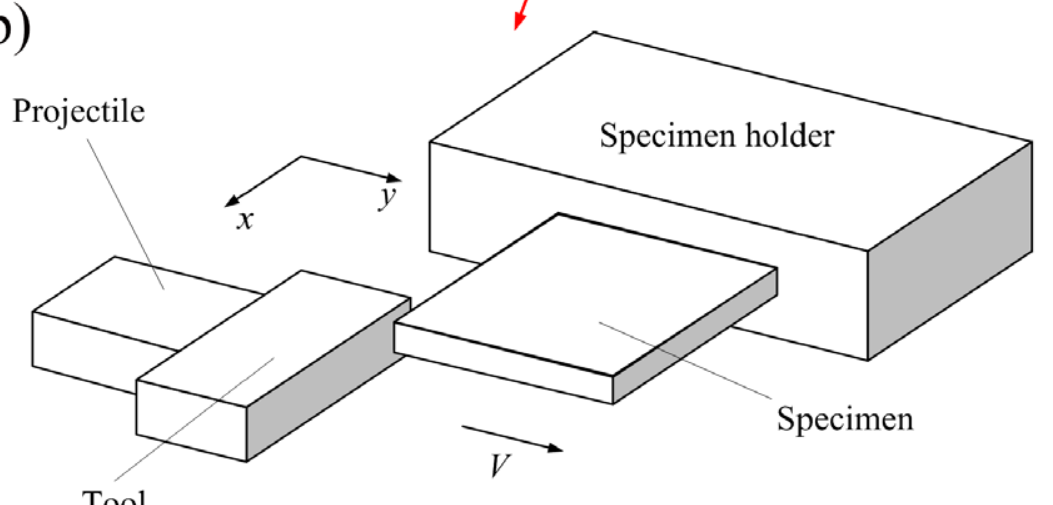

Figure 2. (a) Illustration of the experiment setup based on a split Hopkinson bar and (b) the tool and specimen holder.

\section{Theoretical analysis}

\subsection{Nonlinear dynamic model}

To build up a simple and effective model, let us first consider the modelling of PSZ. As shown in figure 6, two highly localized zones (PSZ and SSZ) carry nearly all the deformation in a cutting process [22]. Thus, it is assumed that deformation only occurs in local zones of PSZ and SSZ.

As illustrated in figure 7(a), the length of PSZ is marked as $l_{1}$, the width of PSZ is $h$ and $w$ is the width of a chip perpendicular to the schematic. The equilibrium equation along the shear direction can be represented as

$$
\int_{0}^{\tau_{1}} l_{1} \mathrm{~d} \tau_{1}=\int_{0}^{h}\left[\sigma_{2} \cos (\theta-\alpha)+\tau_{2} \sin (\theta-\alpha)\right] \mathrm{d} y,
$$

where $\theta$ is the shear angle, $\alpha$ is the tool rake angle, $\sigma_{2}$ is the compressive stress caused by the cutting tool, $\tau_{1}$ is the shear stress in PSZ, and $\tau_{2}$ is the shear stress at the tool tip. As shown in figure 7(a), the shear stress $\tau_{1}$ in PSZ is believed as a linear distribution [23], because near the un-deformed region, there is no shear stress. While on the top of PSZ, shear stress builds up to its maximum due to the high $\sigma_{2}$ near the tool tip zone. Here, friction in SSZ is divided into two parts: sticking and sliding zones. Near the tool tip region, friction is assumed as static, and in the sliding zone, it can be modelled by Bowden and Tabor's theory [24]. So the friction 


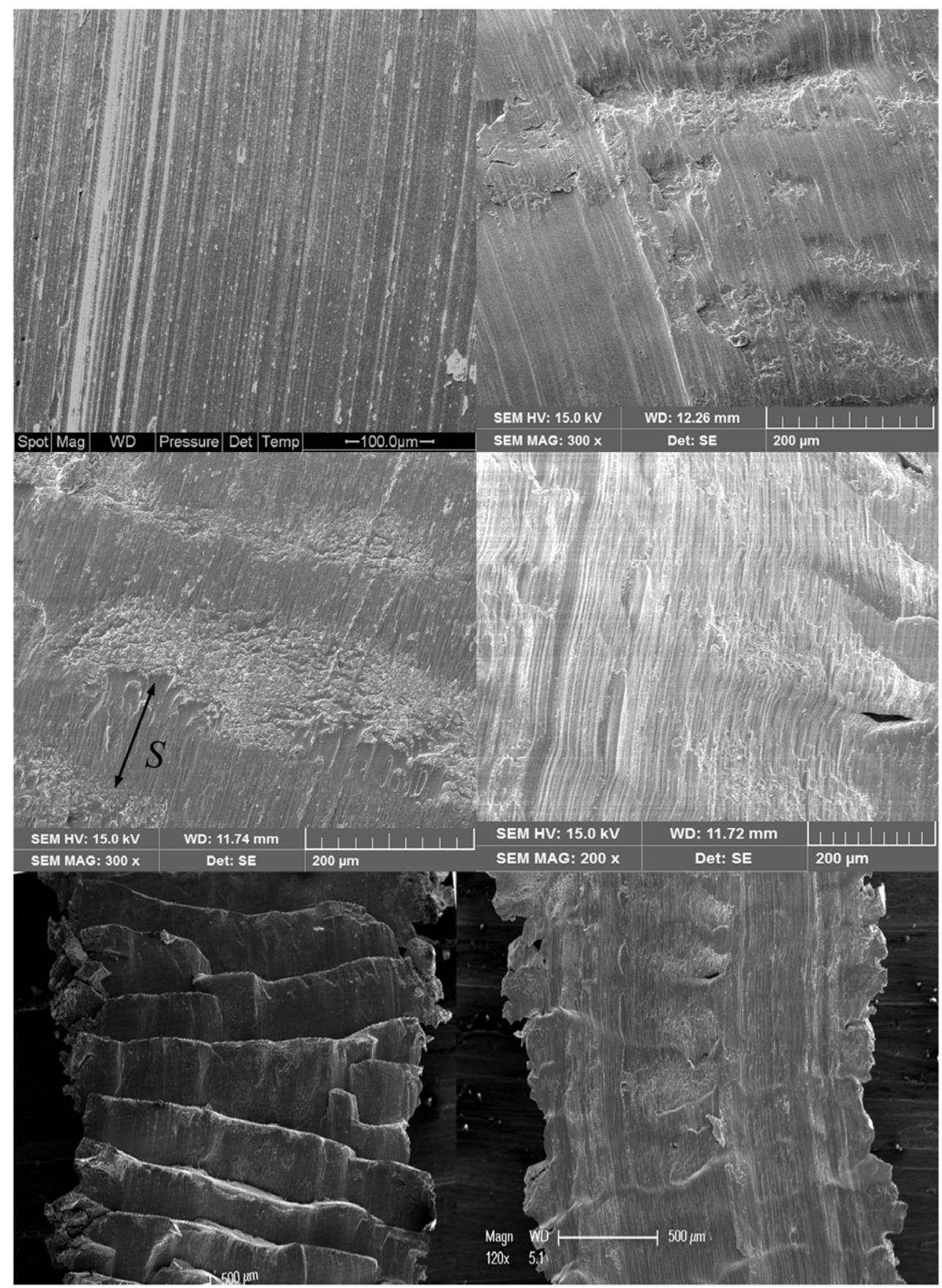

Figure 3. Friction patterns on the tool-chip interface at different cutting speeds of (a) 0.8 , (b) 3, (c) 5, and (d) $10 \mathrm{~m} \mathrm{~s}^{-1}$, where slip length is marked with black arrow. (e) The comparison between the saw teeth on the free surface and stick-slip friction traces on the tool-chip interface. 

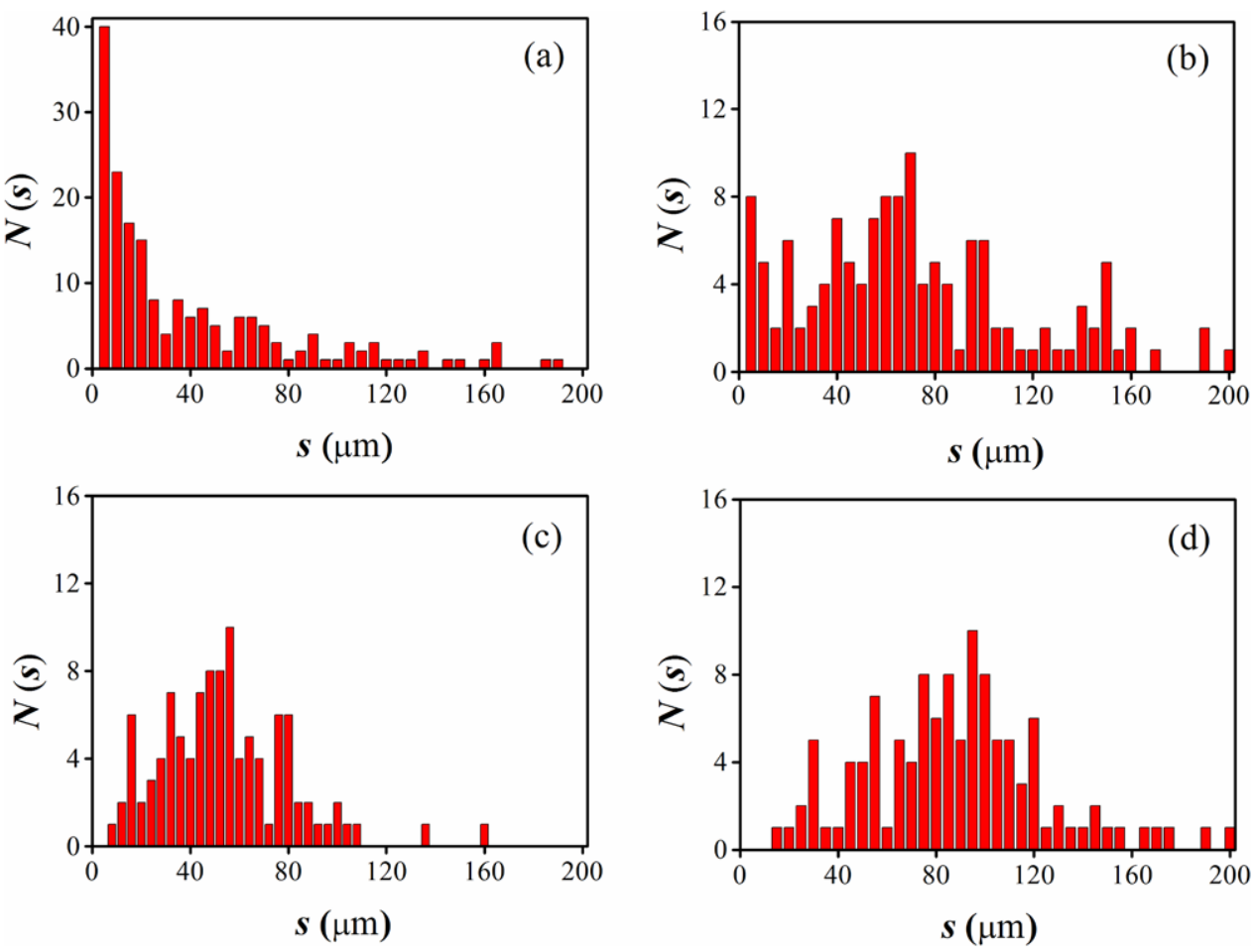

Figure 4. Distribution of the slip number $N(s)$ with the slip-length of $s$ at different cutting speeds of (a) 3 , (b) 5 , (c) 7 , and (d) $10 \mathrm{~m} \mathrm{~s}^{-1}$.

condition at the tool tip can be presented as $\tau_{2}=\mu_{s} \sigma_{2}$, where $\mu_{s}$ is the static friction coefficient. Then, based on the local deformation zones developed by Burns and Davies [25], the shear stress evolution is obtained by the time differentiation of the equilibrium equation, that is

$$
\frac{\mathrm{d} \tau_{1}}{\mathrm{~d} t}=\left[\cos (\theta-\alpha)+\mu_{s} \sin (\theta-\alpha)\right] E \frac{V-\dot{\gamma}_{1} h}{l_{1}},
$$

where $E$ is the elastic modulus, $V$ is the cutting speed, and $\dot{\gamma}_{1}$ is the shear strain rate. Here, the compressive stress $\sigma_{2}$ is induced by the velocity difference between the cutting tool and shear deformation in PSZ [26]. Initially, there is little deformation in a cutting specimen and $\dot{\gamma}_{1}=0$, but $\sigma_{2}$ quickly builds up. Once a shear band is activated in PSZ, i.e. $\dot{\gamma}_{1}>0$, the compressive stress $\sigma_{2}$ starts to unload.

Next, let us focus on the SSZ or the main contact region. As shown in figure 7(b), the motion equation along the sliding direction can be obtained according to the balance of momentum. Here, the problem is how to model the tool-chip tribological condition [27]. According to the Bowden and Tabor's friction model [24], the friction force in SSZ can be characterized as $\tau_{2} A_{r}$, with $\tau_{2}$ the shear stress in SSZ and $A_{r}$ the real contact area. Due to the high normal stress [28], $A_{r}$ is approximately equal to $A$ [29]. Thus, the motion equation can be written as

$$
\rho l_{1} l_{2} w \frac{\mathrm{d}^{2} y}{\mathrm{~d} t^{2}}=\left[\sigma_{1} \cos (\theta-\alpha)-\tau_{1} \sin (\theta-\alpha)\right] l_{1} w-\tau_{2} A,
$$

where $\sigma_{1}$ is the compressive stress in PSZ, which is also induced by the velocity difference and can be calculated by $\sigma_{1}=E(t \cdot V \sin \theta / \cos \alpha-y) / l_{2}, \mathrm{~d}^{2} y / \mathrm{d} t^{2}$ is the acceleration of SSZ, and $\rho$ is 

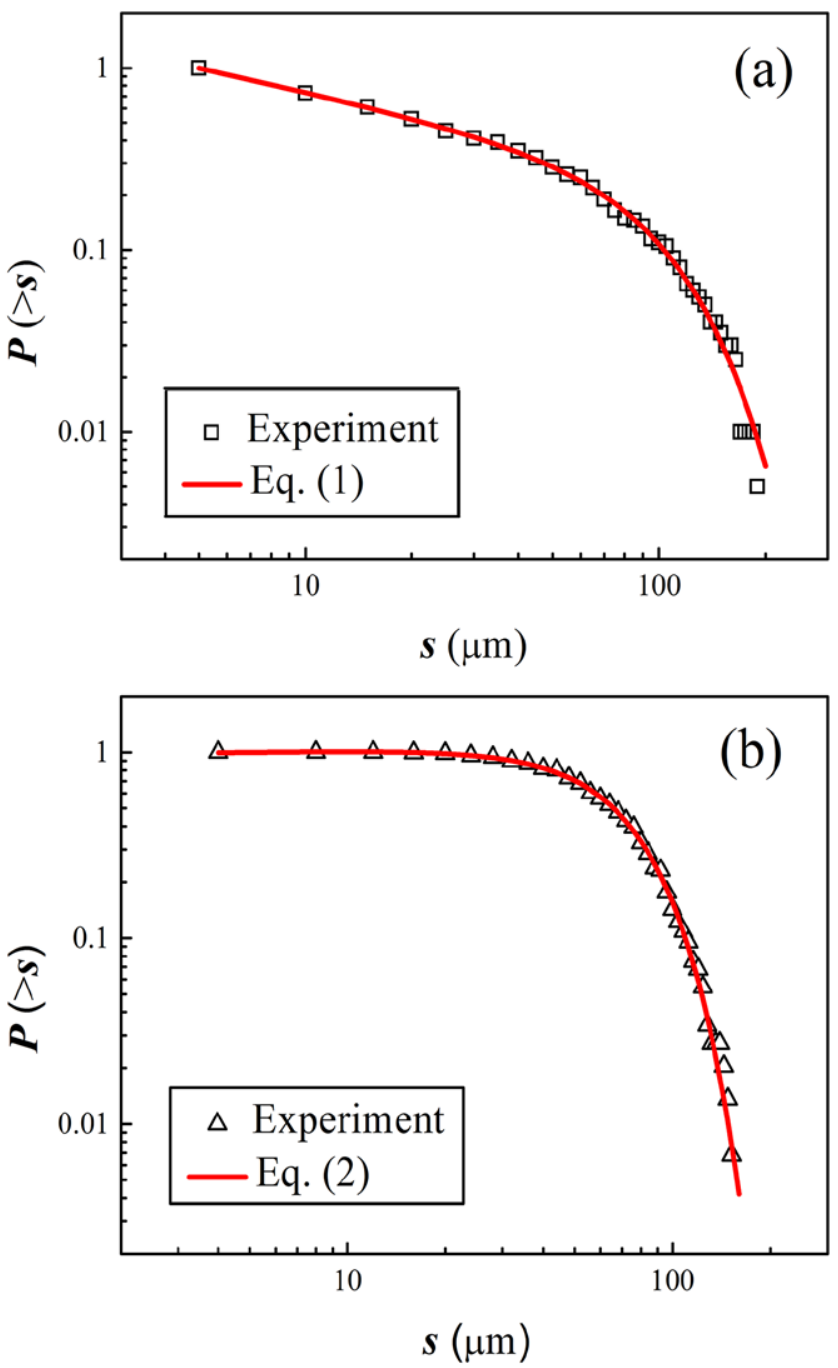

Figure 5. The cumulative probability distribution between the slip number and length at (a) $3 \mathrm{~mm} \mathrm{~s}^{-1}$ and (b) $10 \mathrm{~m} \mathrm{~s}^{-1}$, where the former can be fitted by a power law with an exponential cutoff and its power exponent $\gamma=0.44 \pm 0.02$, and the latter is fitted by an exponential law.

the density of material. In equation (5), the left side represents the inertia term of a chip and its right side is the resultant force.

To describe the temperature evolution of a cutting process, according to the first law of thermodynamics, we have

$$
\Delta \dot{U}=\delta \dot{Q}+\delta \dot{W},
$$

where $\Delta \dot{U}$ is the rise of internal energy per unit time and per unit volume, and $\Delta \dot{U}=\rho c \frac{\mathrm{d} \Delta T}{\mathrm{~d} t}$ with $c$ the specific heat of a material and $\Delta T$ the temperature rise in the control volume. The first term $\delta \dot{Q}$ on the right side of equation (6) represents the heat flux, which is governed by convection and conduction, and the last term $\delta \dot{W}$ is the rate of plastic work. 


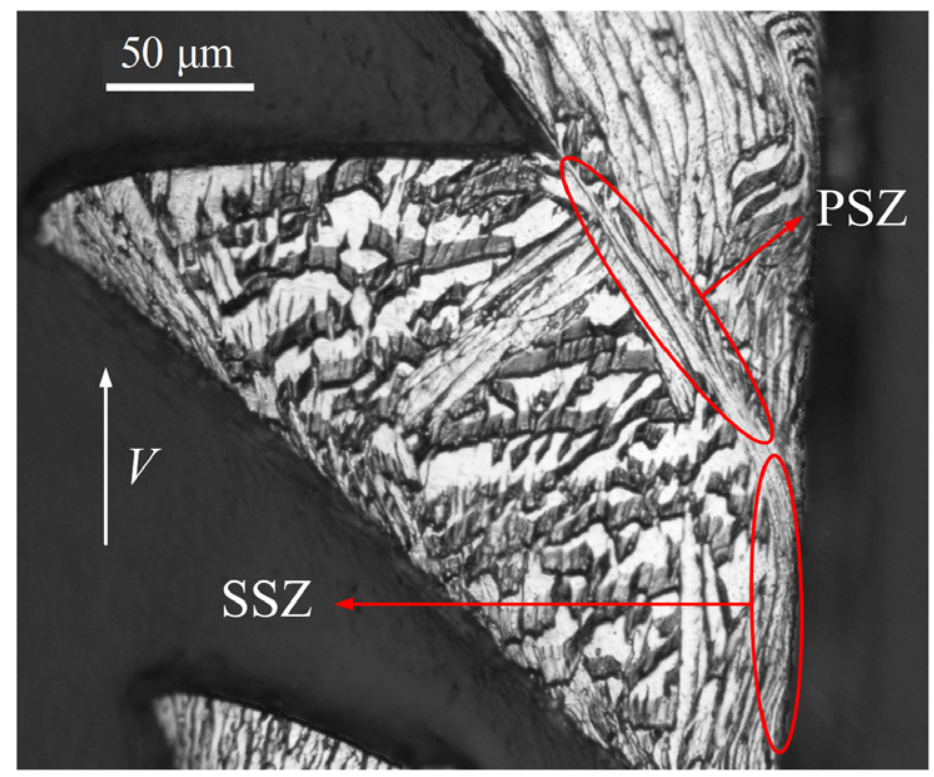

Figure 6. Parts of chip at a cutting speed of $3 \mathrm{~m} \mathrm{~s}^{-1}$, where the highly localized deformation regions (PSZ and SSZ) are marked with red circles. The arrow refers to the chip sliding direction.

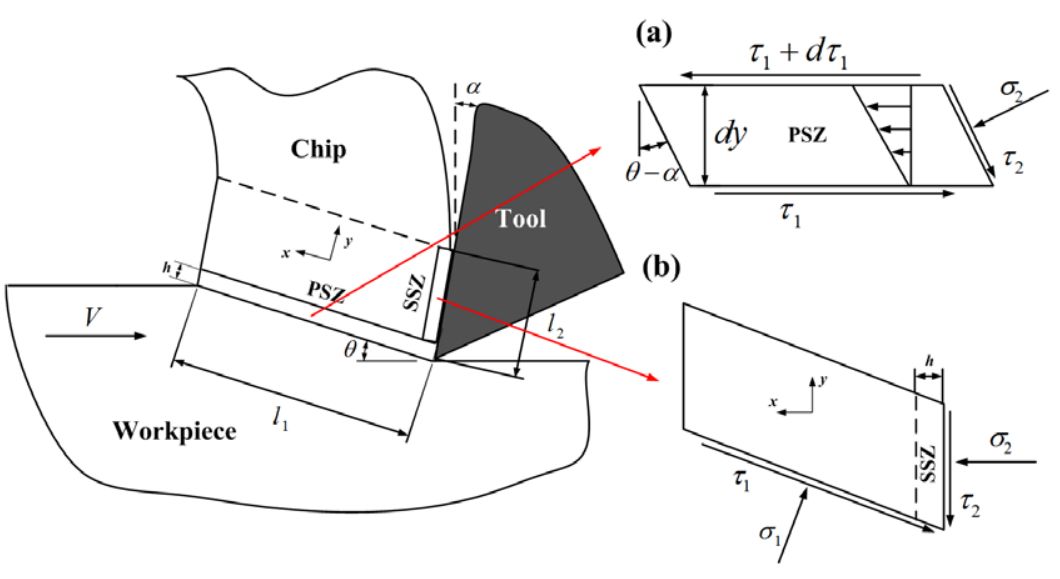

Figure 7. The stress equilibrium diagrams in (a) PSZ and (b) SSZ.

In PSZ, since the shear band occurs in a very narrow region and its nucleation and evolution is fast under high speed cutting condition, the shear process in PSZ is supposed to be adiabatic. So we have $\delta \dot{Q}=0$. Thus, the temperature evolution equation in PSZ can be written as

$$
\rho c \frac{\mathrm{d} T_{1}}{\mathrm{~d} t}=\beta \tau_{1} \dot{\gamma}_{1},
$$

where $T_{1}$ is the temperature in PSZ, and $\beta$ is Taylor-Quinney coefficient (for simplicity, $\beta=1$ is chosen here). The left side of the equation is the internal energy rise in PSZ, while the right side is the plastic work dissipated. 
In contrast to PSZ, the temperature evolution in SSZ is much more complicated. In consideration that SSZ includes a part of PSZ and through it, materials enter into SSZ, the internal energy rise in SSZ is written as $\Delta \dot{U}=\rho c \frac{\mathrm{d}\left(T_{2}-T_{1}\right)}{\mathrm{d} t}$, where $T_{2}$ is the temperature of SSZ. The convection in SSZ induced by chips slides out and takes the heat away from the control volume, which can be approximated by the first order upstream scheme. Due to tiny deformation regions, it can be written as $-2 \rho c V \frac{T_{2}-T_{0}}{h} \frac{\sin \theta}{\cos \alpha}$, where $h$ is the width of SSZ, which is assumed as the same order of PSZ. Based on the central difference scheme, the heat conduction that is governed by the Fourier's law has the form of $-8 \kappa \frac{T_{2}-T_{0}}{h^{2}}$, with $T_{0}$ the temperature in a nearby un-deformed area and $\kappa$ the thermal conductivity. The negative sign of these two terms indicates that heat flows out of the control volume since the deformed area is a high temperature heat source. The plastic work induced by friction can be represented as $\delta \dot{W}=\tau_{2} \dot{\gamma}_{2}$, where $\dot{\gamma}_{2}$ is the equivalent shear strain rate in SSZ that is defined as $\dot{y} / h$, with $\dot{y}$ the sliding speed. Finally, the energy equation of SSZ can be written as

$$
\rho c \frac{\mathrm{d}\left(T_{2}-T_{1}\right)}{\mathrm{d} t}=-2 \rho c V \frac{T_{2}-T_{0}}{h} \frac{\sin \theta}{\cos \alpha}-8 \kappa \frac{T_{2}-T_{0}}{h^{2}}+\tau_{2} \dot{\gamma}_{2} .
$$

Here, it is worth noting that the same as PSZ, all the plastic work is assumed to convert into heat in SSZ.

The plastic flow under a high strain rate is a complicated and thermo-mechanical coupled behaviour [30]. For simplicity, we only consider strain-rate hardening and thermo-softening effects in the constitutive relationship [31], that is

$$
\tau=\tau_{y}\left(1+a \ln \frac{\dot{\gamma}_{2}}{\dot{\gamma}_{0}}\right)\left(1-\bar{T}_{2}\right),
$$

where $\tau_{y}$ is the yield stress, $a$ is the strain-rate hardening coefficient, $\dot{\gamma}_{0}$ is the reference strain rate, and $\bar{T}$ is the dimensionless temperature defined as $\left(T_{2}-T_{0}\right) /\left(T_{\mathrm{m}}-T_{0}\right)$, with $T_{\mathrm{m}}-T_{0}$ the reference temperature. Here $T_{\mathrm{m}}$ and $T_{0}$ are the specimen melting temperature and un-deformed area temperature, respectively.

Based on equations (5) - (8), a set of governing equations of a total cutting process can be obtained. After simplification, the governing equations are

$$
\begin{aligned}
& \rho l_{1} l_{2} w \frac{\mathrm{d}^{2} y}{\mathrm{~d} t^{2}}=\left[\frac{E(t \cdot V \sin \theta / \cos \alpha-y)}{l_{2}} \cos (\theta-\alpha)-\tau_{1} \sin (\theta-\alpha)\right] l_{1} w-\tau_{2} A, \\
& \frac{\mathrm{d} \tau_{1}}{\mathrm{~d} t}=\left[\cos (\theta-\alpha)+\mu_{s} \sin (\theta-\alpha)\right] E \frac{V-\dot{\gamma}_{1} h}{l_{1}}, \\
& \rho c \frac{\mathrm{d} T_{2}}{\mathrm{~d} t}=-2 \rho c V \frac{T_{2}-T_{0}}{h} \frac{\sin \theta}{\cos \alpha}-8 \kappa \frac{T_{2}-T_{0}}{h^{2}}+\left(\tau_{2} \dot{\gamma}_{2}+\tau_{1} \dot{\gamma}_{1}\right) .
\end{aligned}
$$

To make these equations self-consistent, a geometrical condition is needed. According to the velocity diagram, shear and sliding velocities have the relationship of $V_{\text {sliding }}=V_{\text {shear }} \sin \theta / \cos \alpha$ [32]. Thus, the strain rate consistent condition is

$$
\dot{\gamma}_{2}=\dot{\gamma}_{1} \frac{\sin \theta}{\cos \alpha} \text {. }
$$




\subsection{Linear stability analysis}

To investigate the stability of a cutting system, let us introduce the following dimensionless variables

$$
\hat{\tau}_{1}=\frac{\tau_{1}}{\tau_{y}}, \hat{\tau}_{2}=\frac{\tau_{2}}{\tau_{y}}, \hat{T}_{2}=\frac{T_{2}-T_{0}}{T_{\mathrm{m}}-T_{0}}, \hat{t}=\frac{E V}{\tau_{y} l_{1}} t, \hat{y}=\frac{y}{l_{2}}, \hat{\dot{\gamma}_{1}}=\frac{\dot{\gamma}_{1}}{V / h} \text { and } \hat{\dot{\gamma}_{2}}=\frac{\dot{\gamma}_{2}}{V / h}
$$

Considering the real cutting conditions in experiments, the tool rake angle is $\alpha=0^{\circ}$. Then we can rewrite the governing equations (10) - (12) in their non-dimensional forms as

$$
\begin{aligned}
& \frac{\mathrm{d}^{2} \hat{y}}{\mathrm{~d} \hat{t}^{2}}=\frac{\hat{t}-\hat{y}}{\mathrm{Da}} \cos \theta-\frac{\hat{\tau}_{1}}{\mathrm{Da}} \sin \theta-\frac{\hat{\tau}_{2}}{\mathrm{Da}}, \\
& \frac{\mathrm{d} \hat{\tau}_{1}}{\mathrm{~d} \hat{t}}=m\left(1-\hat{\dot{\gamma}_{1}}\right), \\
& \frac{\mathrm{d} \hat{T_{2}}}{\mathrm{~d} \hat{t}}=-2 \sin \theta \cdot \xi \hat{T}_{2}-\frac{8 \xi}{\mathrm{Pe}} \hat{T}_{2}+\xi \eta\left(\hat{\tau_{1}} \hat{\dot{\gamma}_{1}}+\hat{\tau}_{2} \hat{\dot{\gamma}}_{2}\right) .
\end{aligned}
$$

Here, the governing equations have a steady-state solution in the case of

$$
\frac{\mathrm{d} \hat{y}}{\mathrm{~d} \hat{t}}=\frac{\mathrm{d} \hat{\tau}_{1}}{\mathrm{~d} \hat{t}}=\frac{\mathrm{d} \hat{T}_{2}}{\mathrm{~d} \hat{t}}=0
$$

and their corresponding steady state solutions are indicated as $y^{*}, \tau_{1}^{*}$ and $T_{2}^{*}$. Introducing a small perturbation to their solutions, that is

$$
\left(\begin{array}{c}
\hat{y} \\
\hat{\tau}_{1} \\
\hat{T}_{2}
\end{array}\right)=\left(\begin{array}{c}
y^{*} \\
\tau_{1}^{*} \\
T_{2}^{*}
\end{array}\right)+\left(\begin{array}{c}
\delta y \\
\delta \tau_{1} \\
\delta T_{2}
\end{array}\right) \mathrm{e}^{\lambda t},
$$

and retaining the linear terms, we can obtain a linearization governing equation as

$$
J\left(\begin{array}{l}
\hat{y} \\
\hat{\tau}_{1} \\
\hat{T}_{2}
\end{array}\right)=0,
$$

where $J$ is the Jacobian matrix, with the form of

$$
J=\left(\begin{array}{ccc}
\lambda^{2}+\frac{\cos \theta}{\mathrm{Da}} & \frac{\sin \theta}{\mathrm{Da}} & \frac{1}{\mathrm{Da}} \frac{\partial \tau_{2}}{\partial T_{2}} \\
+\frac{1}{\mathrm{Da}} \frac{\partial \hat{\tau}_{2}}{\partial \hat{\dot{\gamma}}_{2}} & \alpha & 0 \\
\frac{m}{\sin \theta} & \lambda+2 \xi \sin \theta+\frac{8 \xi}{\mathrm{Pe}} \\
-\lambda \eta \xi\left(\begin{array}{c}
\frac{\tau_{1}^{*}}{\sin \theta}+\tau_{2}^{*} \sin \theta \\
+\sin \theta \frac{\partial \hat{\tau}_{2}}{\partial \hat{\dot{\gamma}}_{2}}
\end{array}\right)-\frac{\eta \xi}{\sin \theta} & -\eta \xi \sin \theta \frac{\partial \hat{\tau}_{2}}{\partial \hat{T}_{2}}
\end{array}\right) .
$$


In order to have a nontrivial solution, the determinant of $J$ must be equal to zero. Then, a fourth-order eigenvalue equation can be obtained as

$$
\lambda^{4}+a_{1} \lambda^{3}+a_{2} \lambda^{2}+a_{3} \lambda+a_{4}=0,
$$

and its coefficients, $a_{1}, a_{2}, a_{3}$, and $a_{4}$, are

$$
\begin{aligned}
& a_{1}=2 \xi \sin \theta+\frac{8 \xi}{\mathrm{Pe}}-\eta \xi \sin \theta \frac{\partial \hat{\tau}_{2}}{\partial \hat{T}_{2}}, \\
& a_{2}=\frac{\cos \theta}{\mathrm{Da}}+\frac{1}{\mathrm{Da}} \frac{\partial \hat{\tau}_{2}}{\partial \hat{\dot{\gamma}}_{2}}+\frac{\xi \eta}{\mathrm{Da}} \frac{\partial \hat{\tau}_{2}}{\partial \hat{T}_{2}}\left[\left(\frac{\partial \hat{\tau}_{2}}{\partial \hat{\dot{\gamma}}_{2}}+\tau_{2}\right) \sin \theta+\frac{\tau_{1}}{\sin \theta}\right], \\
& a_{3}=-\frac{m}{\mathrm{Da}}+\left(\frac{\cos \theta}{\mathrm{Da}}+\frac{1}{\mathrm{Da}} \frac{\partial \hat{\tau}_{2}}{\partial \hat{\dot{\gamma}}_{2}}\right)\left(2 \xi \sin \theta+\frac{8 \xi}{\mathrm{Pe}}-\eta \xi \sin \theta \frac{\partial \hat{\tau}_{2}}{\partial \hat{T}_{2}}\right),
\end{aligned}
$$

and

$$
a_{4}=-\frac{m}{\mathrm{Da}}\left[2 \xi \sin \theta+\frac{8 \xi}{\mathrm{Pe}}-\left(\sin \theta-\frac{1}{\sin ^{2} \theta}\right) \eta \xi \frac{\partial \hat{\tau}_{2}}{\partial \hat{T}_{2}}\right] .
$$

If an eigenvalue $\lambda$ exists with its real part $\operatorname{Re}(\lambda)>0$, the solution is unstable. According to the Routh-Hurwitz criterion [33], the stable conditions are

$$
a_{1}>0, a_{3}>0, a_{4}>0 \text {, and } a_{1} a_{2} a_{3}>a_{3}^{2}+a_{1} a_{4} .
$$

Thus, the system becomes unstable if one of these four conditions is not satisfied.

Under the present cutting situation, the unstable condition is $a_{3}<0$. Following the magnitude analysis and neglecting infinite small terms, a relatively simple instability criterion (notated by $\mathrm{Cr}$ ) can be obtained as

$$
C r=\frac{\eta \xi \sin \theta \frac{\partial \hat{\tau}_{2}}{\partial \hat{T}_{2}}+\frac{m}{\cos \theta+\frac{\partial \hat{\tau}_{2}}{\partial \hat{\gamma}_{2}}}}{2 \xi \sin \theta+\frac{8 \xi}{P e}}>1,
$$

where $m$ is in the form of

$$
m=\cos \theta+\mu_{s} \sin \theta,
$$

and other dimensionless parameters are respectively as follows:

$$
\xi=\frac{\tau_{y} l_{1}^{2}}{E l_{2} h}, \eta=\frac{\tau_{y}}{\rho c\left(T_{\mathrm{m}}-T_{0}\right)}, \mathrm{Pe}=\frac{V h}{\lambda} \text { and } \mathrm{Da}=\frac{\rho V^{2}}{\tau_{y}},
$$

where $\lambda=\kappa / \rho c$ is the thermal diffusivity, $\xi$ is the ratio between shear and compressive forces, $\eta$ is the heat generation number, Pe is the Peclet number defined as the ratio of thermal convection to diffusion, and $\mathrm{Da}$ is the damage number of the external dynamic pressure dividing by the resistant stress.

To examine the validity of this instability criterion, the mechanical properties of Ti-Al6-V4 were utilized in theoretical analysis. For such a titanium alloy, $\rho=4430 \mathrm{~kg} \mathrm{~m}^{-3}, c=620 \mathrm{~J} \mathrm{~K}^{-1}$, $\kappa=6.6 \mathrm{~W} /(\mathrm{m} \cdot \mathrm{K})$, the melt temperature $T_{\mathrm{m}}=1783 \mathrm{~K}$, and the un-deformed material temperature $T_{0}=293 \mathrm{~K}$. Other constitutive parameters are given as follows: the elastic modulus $E=110 \mathrm{GPa}$, yield stress $\tau_{y}=782 \mathrm{MPa}$, reference strain rate $\dot{\gamma}_{0}=10^{-5}$, and the strain hardening 


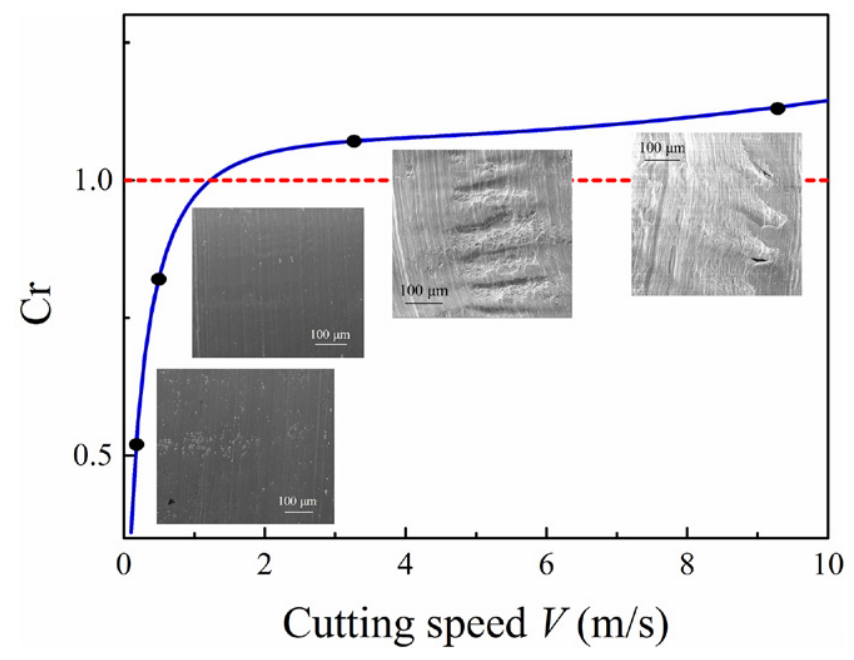

Figure 8. The instability criterion for Ti-6Al-4V, where insets are experimental observations at various speeds indicated by black dots.

coefficient $a=0.028$ [34]. Based on the instability criterion in equation (28), as shown in figure 8 , the transition point from stable to unstable states is around a cutting speed of $1.0 \mathrm{~m} \mathrm{~s}^{-1}$, which is consistent with experimental observations (see insets in figure 8).

For a given material, $\xi$ and $\eta$ are constants whereas the other parameters are dependent on the cutting speed in experiments. Thus, it is found from equation (28) that the main promoting factors of instability are friction thermal softening effects, i.e. $\partial \hat{\tau}_{2} / \partial \hat{T}_{2}$, in SSZ, and the parameter $m$, which indicates the cutting geometric configuration and static friction influences. The resistant factors include the rate-hardening effect of frictional stress such as $\partial \hat{\tau}_{2} / \partial \hat{\gamma}_{2}$ and the convection and diffusion of heat, which is involved in the Peclet number Pe. As the increase of cutting speed, however, Pe may also play a promoting factor because the total denominator of $\mathrm{Cr}$ becomes smaller. This is the reason why instability always occurs at a relative high cutting speed.

\subsection{Numerical simulation}

By using the Runge-Kutta method and MATLAB ODE23s solver, numerical simulations of governing equations were carried out.

The results indicate that the phase diagrams, as shown in figure 9 , are well consistent with the experiments (see figure 3). The cutting process is stable at velocities less than the transition speed, and the corresponding topological structure is a point in the phase space. At a cutting speed of $0.8 \mathrm{~m} \mathrm{~s}^{-1}$, the smooth interface is observed in experiments (see figure 3(a)), which implies a steady sliding process. However, once the cutting speed exceeds the transition point, the dynamic behaviour becomes very complex with a few large and many small stickslip events. As the cutting speed keeps increasing, the slip length becomes regular, similar to that in figure 3(d), which is corresponding to the limit cycle behaviour (see figure $9(\mathrm{~d})$ ). Furthermore, based on the statistical analysis on numerical results, the cumulative slip-length distribution at the speed of $3 \mathrm{~m} \mathrm{~s}^{-1}$, as shown in figure 10, can be also described by a power law with the exponential cutoff as in equation (1), that is 

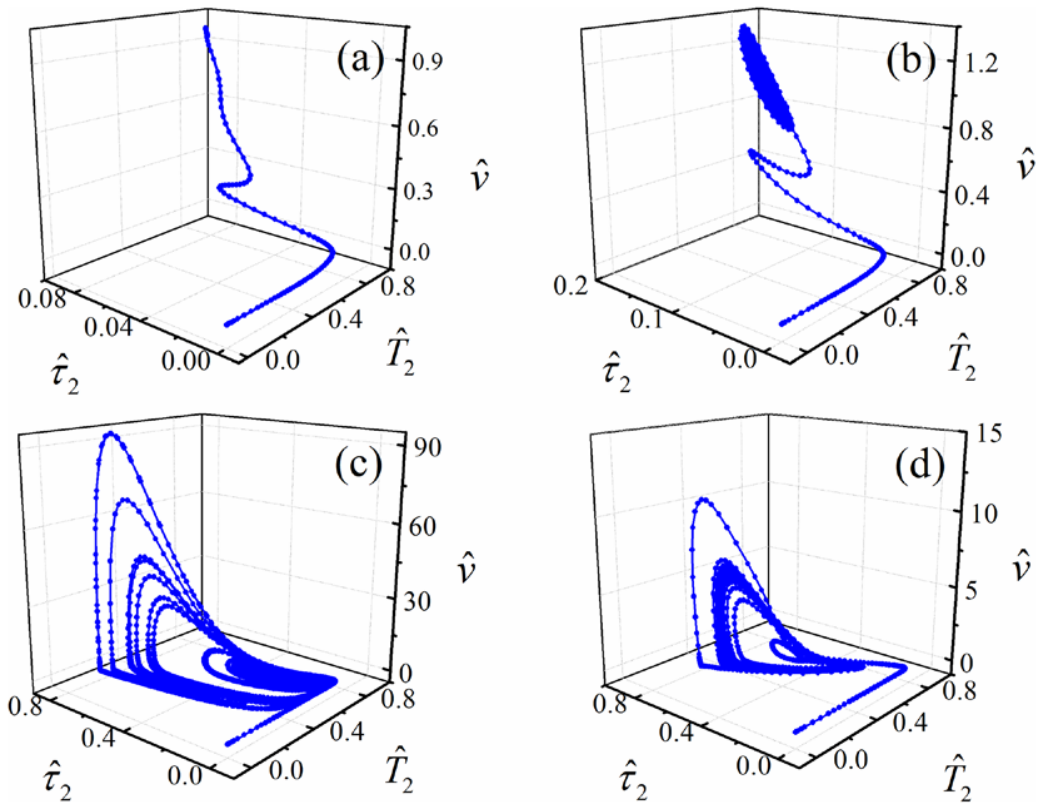

Figure 9. Phase diagrams of a cutting system as the increase of cutting speeds, where the $\hat{v}=\mathrm{d} \hat{y} / \mathrm{d} \hat{t}$ represents the dimensionless sliding speed of SSZ: (a) 0.5, (b) 1.1, (c) 3, and (d) $10 \mathrm{~m} \mathrm{~s}^{-1}$.

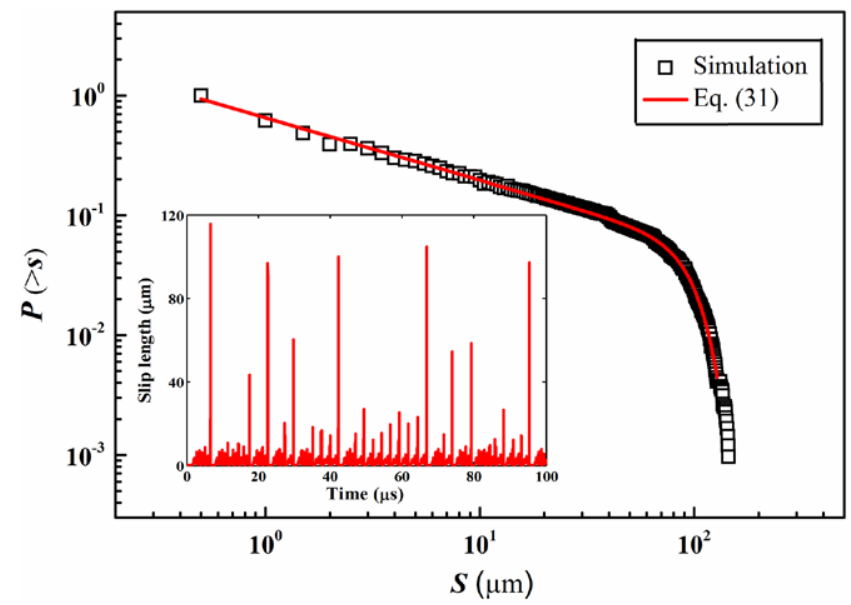

Figure 10. Numerical and experimental results of the cumulative probability distribution at a cutting speed of $3 \mathrm{~m} \mathrm{~s}^{-1}$, the slope of linear part in these power law scaling are almost the same. Inset shows the change of slip length versus time.

$P(>s) \infty s^{-\alpha} \exp \left(-s / s^{*}\right)$,

with the power law exponent $\alpha=0.49 \pm 0.01$ and the characteristic length $s^{*}=100.4 \pm 1.1 \mu \mathrm{m}$, which is in agreement with the experimental results at the same cutting speed. 
Metal cutting is spatio-temporally correlated, which is the same as many other complex systems. To have a comprehensive understanding of the friction behaviour in metal cutting, it is necessary to investigate the temporal evolution. Previous works has shown that, although its temporal evolution is difficult to be extracted in experiments, a sliding event can be considered as the indication of dissipation [35]. In simulations, we can obtain a time series of sliding events, as shown in the inset of figure 10. Here, the sliding length is calculated by an integration of a sliding speed versus its time. In terms of the Fourier transformation, the power spectrum of a sliding time series is

$$
S(f)=\int\langle l(t+\Delta t) l(t)\rangle \exp (2 \pi \mathrm{i} f \Delta t) \mathrm{d} \Delta t
$$

where $l(t)$ is the sliding length at the time of $t, f$ is the frequency, $\Delta t$ is the time lag and the square bracket represents an ensemble average over the total length of a time series. The results in figure 11(a) show that the power spectrum also follows a power law with the exponent of 1.82 , which is similar to that obtained from the dry-friction signals of M50 steel [36].

It is worth noting, however, as velocity increases, the cutting system gradually behaves like a periodic oscillation, which corresponds to a limit cycle in the phase diagram. The power spectrum at a higher cutting speed, as shown in figure 11(b), has a peak-characteristic (or single dominated) frequency as expected, which is in accordance with the limit cycle behaviour. That means such a critical state only appears in a narrow scope of cutting speeds. That is, the cutting velocity is the key tuning parameter in stick-slip friction. In contrast to the self-organized criticality, the cutting speed (or cutting geometrical configuration) must be tuned into a critical value to obtain the power law distribution. Given that the tuning parameter is changed, dynamical characteristics of the system may transfer to other behaviours [37].

\section{Discussion}

The transition from a speed-tuned criticality state to a limit cycle is an interesting phenomenon. Based on the above-mentioned model, it is obvious that, due to the coupled shear and friction, there are two characteristic times in a cutting system. One is the unloading time of a shear band and the other is the inertia time to describe the motion in the $y$-axis direction.

As shown in figure 12, the average period of a speed-tuned critical state is between these two characteristic times. As the cutting speed increases, the average period finally coincides with the inertia time. That is, the system is governed by its inertia effect at a very high cutting speed, which is similar to the 'frequency lock' phenomenon in a forced Duffing oscillator [38]. The dimensionless parameter Da plays an important role in this secondary transition.

There are a lot of similarities between the friction-tuned criticality and crack front propagation processes. As shown in figure 3, there are two stages, i.e. slow stress build-up and fast sliding in the friction process, which resembles the pinning and de-pinning regions in crack initiation, respectively. The pining size distribution also follows a power law. In crack front propagation experiments [6], however, the scaling relation is independent of the loading conditions, which makes the crack opening more likely a self-organized criticality.

It is also of interest to note that the mechanism of cutting friction dynamics is similar to fault gouge dynamics in earthquakes [39]. If we consider temperature as a state variable, the constitutive relationship of equation (8) is a general form of the rate- and state-dependent friction law [40]. The nonlinear dynamics in earthquake spring-block models are caused 

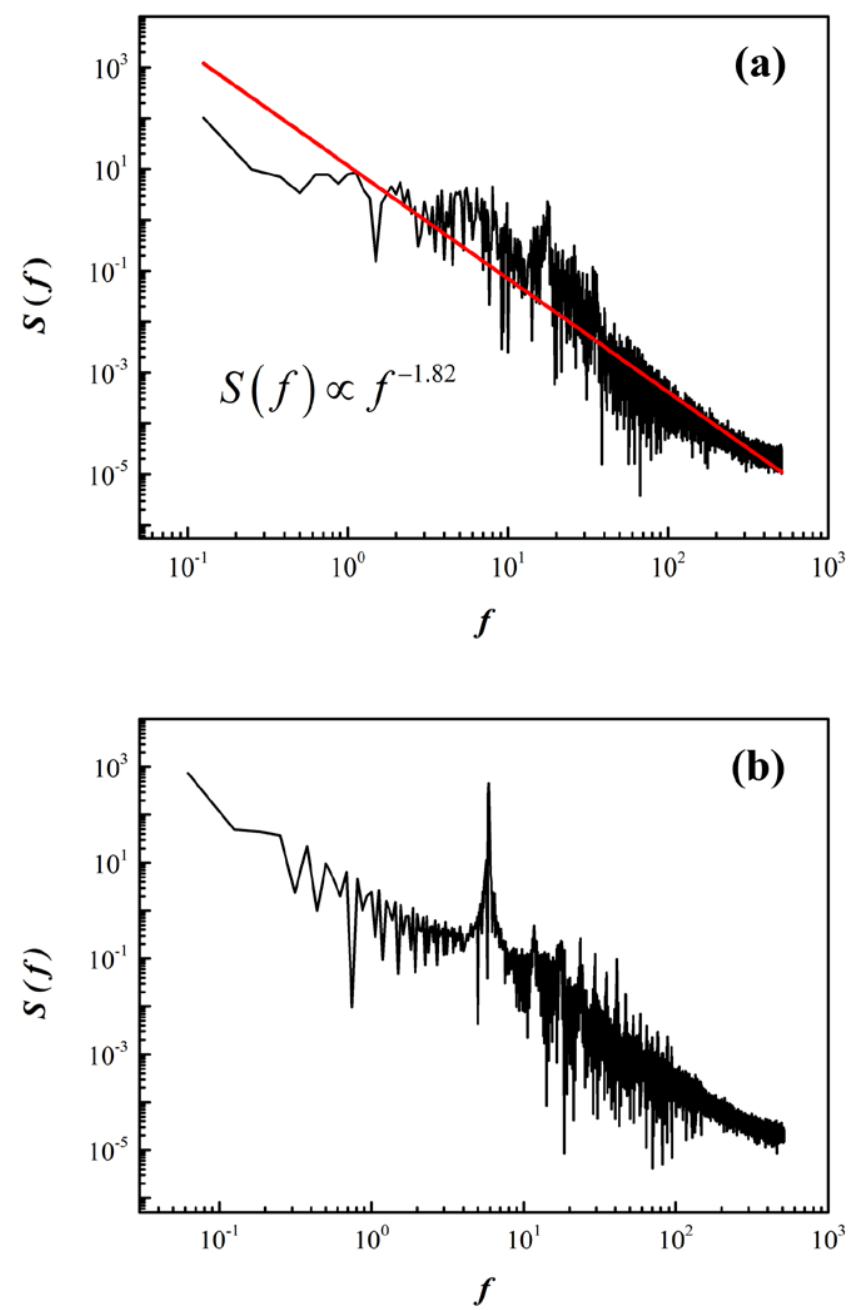

Figure 11. The power spectrums at the cutting speed of (a) $3 \mathrm{~m} \mathrm{~s}^{-1}$ and (b) $10 \mathrm{~m} \mathrm{~s}^{-1}$.

by multiple interactions of stick-slip, and similarly, it is due to the interaction of shear and friction in metal cutting. Because of its sensitivity to perturbation, such a speed-tuned criticality state may bring a negative influence on a metal cutting process, such as irregular tool vibration.

\section{Conclusion}

In conclusion, the experimental and theoretical studies have been performed on a metal cutting friction process. It is shown that, as the increase of cutting speed, metal cutting naturally evolves to a tuned critical state, with a power law distribution of stick-slip events. Based on a nonlinear dynamic model, we find out that the speed-tuned criticality is due to the thermoactivated instability induced by the interaction between shear and friction, which is further transformed into a limit cycle. This research may bring some new insights into the optimal design of parameters such as speed in metal cutting. 


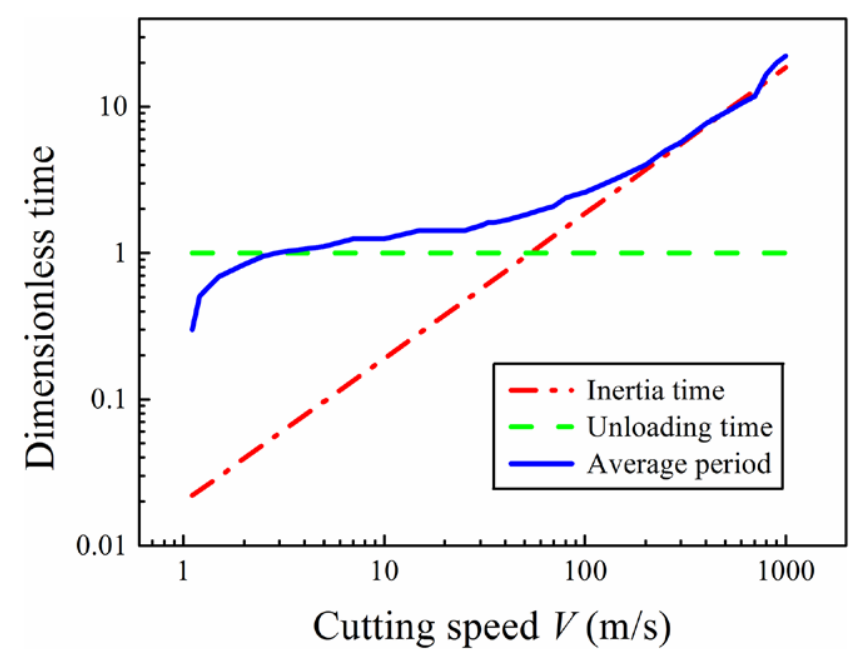

Figure 12. The dimensionless characteristic time versus speed in a cutting process.

\section{Acknowledgments}

This work was supported by the National Natural Science Foundation of China (Grant Nos. 11132011 and 11402278), the National Key Basic Research Program of China (Grant No. 2012CB937500), China Postdoctoral Science Foundation (Grant No. 2013M540148), the CAS/SAFEA International Partnership Program for Creative Research Teams and the Opening Fund of State Key Laboratory of Nonlinear Mechanics. C.L. acknowledges Earthquake Research Institute at the University of Tokyo for the support under its visiting fellowship scheme, and T. Hatano and T. Koyaguchi for helpful discussions.

\section{References}

[1] Atkins T 2009 The Science and Engineering of Cutting: The Mechanics and Processes of Separating and Puncturing Biomaterials, Metals and Non-metals (Amsterdam: Elsevier)

[2] Jackson M J, Whitfield M, Robinson G M, Morrell J and Davim J P 2013 Tribology in Manufacturing Technology ed J P Davim (Berlin: Springer) pp 67

[3] Wiercigroch M and Budak E 2001 Phil. Trans. R. Soc. 359663

[4] Atkins A G 2003 Int. J. Mech. Sci. 45373

[5] Komanduri R and Brown R H 1981 J. Eng. Ind. 10333

[6] Rusinek R, Wiercigroch M and Wahi P 2014 Int. J. Mech. Sci. 89167

[7] Goodman R and Sundaram P N 1978 Pure Appl. Geophys. 116873

[8] Baumberger T and Caroli C 2006 Adv. Phys. 55279

[9] Daub E G and Carlson J M 2009 Phys. Rev. E 80066113

[10] Ye G G, Xue S F, Jiang M Q, Tong X H and Dai L H 2013 Int. J. Plast. 4039

[11] Cai S L and Dai L H 2014 J. Mech. Phys. Solids 7384

[12] Bahi S, Nouari M, Moufki A, El Mansori M and Molinari A 2012 Wear 28645

[13] Wedberg D, Svoboda A and Lindgren L E 2012 Modelling Simul. Mater. Sci. Eng. 20085006

[14] Ozlu E, Molinari A and Budak E 2010 Mach. Sci. Tech. 14323

[15] Tallakstad K T, Toussaint R, Santucci S, Schmittbuhl J and Måløy K J 2011 Phys. Rev. E 83046108

[16] Lu C, Mai Y W and Shen Y G 2005 Phys. Rev. E 72027101

[17] Biswas S and Chakrabarti B K 2013 Phys. Rev. E 88042112

[18] Antonaglia J, Xie X, Schwarz G, Wraith M, Qiao J, Zhang Y, Liaw P K, Uhl J T and Dahmen K A 2014 Sci. Rep. 44382 
[19] Zypman F R, Ferrante J, Jansen M, Scanlon Kand Abel P 2003 J. Phys.: Condens. Matter. 15 L191

[20] Lu C, Vere-Jones D and Takayasu H 1999 Phys. Rev. Lett. 82347

[21] Ye G G, Xue S F, Ma W, Jiang M Q, Ling Z, Tong X H and Dai L H 2012 Int. J. Mach. Tools Manuf. 561

[22] Komanduri R and Von Turkovich B F 1981 Wear 69179

[23] Joshi S S and Melkote S N 2005 J. Manuf. Sci. Eng. 126679

[24] Bowden F P and Tabor D 2001 The Friction and Lubrication of Solids (Oxford: Clarendon)

[25] Burns T J and Davies M A 1997 Phys. Rev. Lett. 79447

[26] Davies M A and Burns T J 2001 Phil. Trans. R. Soc. 359821

[27] Childs T H C 2006 Wear 260310

[28] Trent E M 1988 Wear 12829

[29] Molinari A, Estrin Y and Mercier S 1999 J. Tribol. 12135

[30] Batra R C and Chen L 2001 Int. J. Plast. 171465

[31] Grady D E 1992 J. Mech. Phys. Solids 401197

[32] Merchant M E 1945 J. Appl. Phys. 16267

[33] Jiang M Q and Dai L H 2009 J. Mech. Phys. Solids 571267

[34] Lee W S and Lin C F 1998 J. Mater. Proc. Tech. 75127

[35] Bak P, Tang C and Wiesenfeld K 1988 Phys. Rev. A 38364

[36] Buldyrev S V, Ferrante J and Zypman F R 2006 Phys. Rev. E 74066110

[37] Dahmen K A, Ben-Zion Y and Uhl J T 2011 Nat. Phys. 7554

[38] Kovacic I and Brennan M J 2011 The Duffing Equation: Nonlinear Oscillators and Their Behaviour (Chichester: Wiley)

[39] Kawamura H, Hatano T, Kato N, Biswas S and Chakrabarti B K 2012 Rev. Mod. Phys. 84839

[40] Ruina A 1983 J. Geophys. Res. Solid Earth 8810359 